\title{
Design of an Open-Ended Coaxial Probe for Broadband, Low-Footprint Nondestructive Characterization of PEC-Backed Materials
}

\author{
Milo W. Hyde IV and Michael J. Havrilla \\ Department of Electrical and Computer Engineering \\ Air Force Institute of Technology \\ Dayton, Ohio 45433 \\ Email: milo.hyde@afit.edu
}

\begin{abstract}
The design of an open-ended coaxial probe for the nondestructive characterization of PEC-backed materials is presented. The design attempts to maximize the measurement bandwidth while limiting the size of the probe flange plate. These design criteria are met by analyzing the theoretical reflection coefficient's behavior versus frequency and the coaxial line's inner radius. Included in this paper are the theoretical derivation of the reflection coefficient, accomplished using Love's equivalence theorem, the parallel-plate Green's function, and the Method of Moments (MoM), and the results of the design analysis. Future work is also discussed.
\end{abstract}

\section{INTRODUCTION}

Open-ended coaxial probes have been the subject of research for the past 20 years. They have been predominately employed in the field of nondestructive material evaluation/characterization, e.g., biological tissues [1], [2], negativeindex materials [3], etc. [4]-[10]. While being very useful for nondestructive inspection/evaluation (NDI/NDE) applications especially at low frequencies (as compared to rectangular or circular waveguide probes), coaxial waveguide probes suffer from two well documented issues, namely, weak field coupling into the material under test (MUT) and reflections from the edges of the coaxial flange plate.

The first issue mentioned above is weak field coupling into the MUT region (see Fig. 1) of the probe. This issue manifests itself as a reflection coefficient $S_{11}$ whose magnitude $\left|S_{11}\right| \approx 1$ and whose phase $\angle S_{11}$ is approximately constant. Note that theoretically $S_{11}$ is different for different MUTs; however, when operating in the weak field coupling regime, this difference is so small (i.e., within the measurement uncertainty) that experimentally it is extremely difficult to differentiate one MUT from another. In other words, dissimilar MUTs become "experimentally identical." This field coupling issue is especially pertinent for PEC-backed measurement geometries as depicted in Fig. 1 and analyzed in this paper. Olmi et al. [4], De Langhe et al. [6], and Baker-Jarvis et al. [9] briefly discuss this limitation and provide an approximate design equation for the coaxial probe, namely, $b-a>\lambda_{\mathrm{MUT}} / 10$ where $b$ is the distance to the surface of the outer conductor (outer radius), $a$ is the distance to the surface of the inner conductor (inner radius), and $\lambda_{\text {MUT }}$ is the wavelength in the MUT. The actual

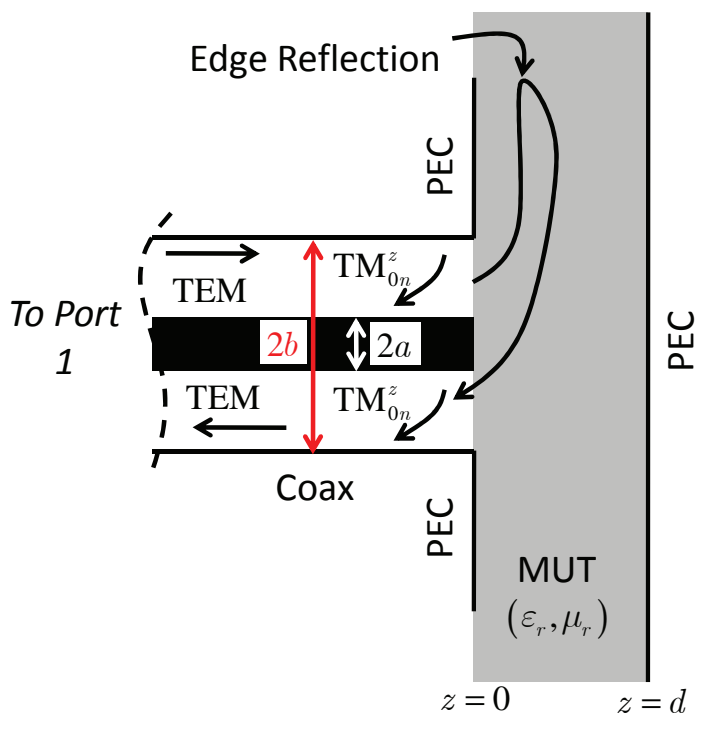

Fig. 1. Open-ended coaxial probe measurement geometry.

amount of field coupling into the MUT region of the probe is a very complex relation involving the MUT's thickness $d$, permittivity $\varepsilon_{r}$, permeability $\mu_{r}$, and the characteristic impedance of the coaxial line $Z_{c}$. This exact analysis is most easily performed in simulation (MoM, FDTD, and FEM) and has historically been done so. It is very important to note that weak field coupling into the MUT region of the probe effectively band limits the measurement between the frequency at which the coupling becomes sufficient (such that MUTs become experimentally distinct) and the cut-off frequency of the first higher-order coaxial waveguide mode (the $\mathrm{TE}_{11}^{z}$ mode).

Assuming one designs their probe well so that the field coupling into the MUT region is sufficient (MUTs become experimentally distinct), reflections from the edges of the coaxial flange will be introduced which will corrupt MUT characterization results (see Figs. 1 and 2). This edge reflection is the second problem encountered when one uses an openended coaxial probe (as well as other open-ended waveguide 


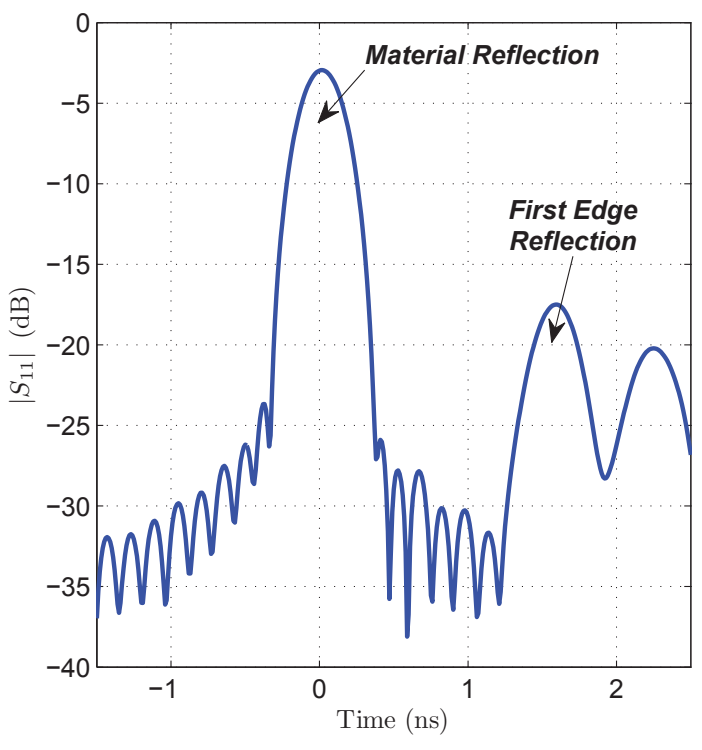

Fig. 2. Time-domain $\left|S_{11}\right|$ in $\mathrm{dB}$ of acrylic measured using an open-ended rectangular waveguide probe with a $30.5 \mathrm{~cm} \times 30.5 \mathrm{~cm}$ aluminum flange.

probe geometries). Olmi et al. [4] and Li et al. [10] both discuss this issue at length. The problem has historically been handled by only measuring very lossy MUTs (so that the propagating wave in the MUT region is sufficiently decayed before reaching the flange edge) or using a very large flange (De Langhe [6] used an $80 \mathrm{~mm}$ diameter circular flange and Li [10] used a $50 \mathrm{~cm}$ by $50 \mathrm{~cm}$ flange). Either of these solutions severely limits the applicability of using an open-ended coaxial probe for nondestructive material characterization.

Recently, Hyde et al. [11] presented a time-domain gating method to remove the edge reflections from open-ended rectangular waveguide probe measurements, thereby permitting such nondestructive geometries to characterize low-loss MUTs and utilize a much smaller flange. To use the method, the first edge reflection (its time location being proportional to the flange size) must be resolved. This requires sufficient measurement bandwidth making broadband measurement field applicators especially attractive.

The purpose of this research is twofold: 1) to determine whether an open-ended coaxial probe is sufficiently broadband (considering the first issue discussed above) to make it a good option as compared to a rectangular waveguide probe (naturally band limited and investigated in [11]) and 2) to present an open-ended coaxial probe design methodology which maximizes measurement bandwidth (i.e., deals specifically with the first issue mentioned above) while limiting flange size (deals with the second issue of open-ended waveguide probes). This paper is organized as follows: Section II presents the theoretical derivation of the reflection coefficient from an open-ended coaxial probe placed against a PEC-backed MUT. The derivation uses Love's equivalence theorem [12] and the parallel-plate Green's function [13] to form a magnetic field integral equation (MFIE) which is subsequently solved using the MoM [14]. Section III presents simulation results for the magnitude and phase of the reflection coefficient for a low-loss MUT (alumina $\mathrm{Al}_{2} \mathrm{O}_{3}$ in this case) versus frequency and inner radius. By examining these plots one can determine both the amount of field coupling into the MUT region of the probe and the measurement bandwidth. Last, a summary of this research is provided including a discussion of future work.

\section{Methodology}

The theoretical expression for the reflection coefficient of the open-ended coaxial probe geometry depicted in Fig. 1 can be found through enforcing the continuity of transverse fields at the probe aperture. In the coax region, the transverse electric and magnetic fields take the form [12]

$$
\begin{aligned}
E_{\rho} & =\frac{1}{\rho}\left[e^{-j k_{c} z}+\Gamma_{\mathrm{TEM}} e^{j k_{c} z}\right] \\
& +\sum_{n=1}^{N} \frac{\Gamma_{n} k_{\rho n} e^{j k_{z n} z}}{\mu_{c}}\left[\frac{Y_{0}\left(k_{\rho n} a\right)}{J_{0}\left(k_{\rho n} a\right)} J_{1}\left(k_{\rho n} \rho\right)-Y_{1}\left(k_{\rho n} \rho\right)\right] \\
H_{\phi} & =\frac{1}{\eta_{c} \rho}\left[e^{-j k_{c} z}-\Gamma_{\mathrm{TEM}} e^{j k_{c} z}\right] \\
& -\sum_{n=1}^{N} \frac{\Gamma_{n} k_{\rho n} e^{j k_{z n} z}}{Z_{n}^{\mathrm{TM}} \mu_{c}}\left[\frac{Y_{0}\left(k_{\rho n} a\right)}{J_{0}\left(k_{\rho n} a\right)} J_{1}\left(k_{\rho n} \rho\right)-Y_{1}\left(k_{\rho n} \rho\right)\right]
\end{aligned}
$$

where $k_{c}=\omega \sqrt{\varepsilon_{c} \mu_{c}}$ is the TEM wave number in the coax, $\eta_{c}$ is the intrinsic impedance of the coax, $k_{\rho n}$ is the $\rho$-directed wave number of the $n^{\text {th }}$ mode, $k_{z n}=\sqrt{k_{c}^{2}-k_{\rho n}^{2}}$ is the $z$ directed wave number of the $n^{\text {th }}$ mode, $Z_{n}^{\mathrm{TM}}$ is the TM coaxial wave impedance, $J_{i}$ and $Y_{i}$ are $i^{\text {th }}$-order Bessel functions of the first kind and second kind, and $\Gamma_{n}$ are the modal reflection coefficients with $\Gamma_{\text {TEM }}$ being the one ultimately desired. Note that the above expressions assume a TEM incident field and only $\phi$-invariant, evanescent higher-order modes are excited at the aperture discontinuity.

In the MUT region, the transverse magnetic field can be found by replacing the probe aperture with an equivalent magnetic current $\mathcal{M}$ in accordance with Love's equivalence theorem. Making use of the dyadic parallel-plate Green's function $\overline{\mathbf{G}}$ [13], the transverse magnetic field in the MUT region of the probe takes the form

$$
\boldsymbol{H}_{\mathrm{MUT}}^{t}=\frac{1}{j \omega \mu \varepsilon}\left(k^{2}+\nabla_{t} \nabla \cdot\right) \iint_{s^{\prime}} \overline{\mathbf{G}} \cdot \varepsilon \mathcal{M} d s^{\prime} .
$$

Having found expressions for the fields in the coax and MUT regions of Fig. 1, an MFIE is derived by enforcing the continuity of the transverse magnetic field at the probe aperture, i.e.,

$$
\begin{aligned}
\hat{\boldsymbol{\phi}} & \cdot \boldsymbol{H}_{\mathrm{MUT}}^{t}(z=0)=\frac{1}{\eta_{c} \rho}\left(1-\Gamma_{\mathrm{TEM}}\right) \\
& -\sum_{n=1}^{N} \Gamma_{n} \frac{k_{\rho n}}{Z_{n}^{\mathrm{TM}} \mu_{c}}\left[\frac{Y_{0}\left(k_{\rho n} a\right)}{J_{0}\left(k_{\rho n} a\right)} J_{1}\left(k_{\rho n} \rho\right)-Y_{1}\left(k_{\rho n} \rho\right)\right]
\end{aligned}
$$

Expression (3) is solved for the modal reflection coefficients using the MoM [14] with the transverse electric field distribution given in (1) serving as the basis set for $\mathcal{M}$ and 
the transverse magnetic field distribution given in (1) serving as the testing set. Note that if the left-hand side of (3) is transformed to the spectral domain, all basis and testing integrals can be computed in closed form. The required inverse Hankel transform is most easily computed using numerical quadrature. The result of this analysis is a matrix equation $\mathbf{A x}=\mathbf{b}$, where $\mathbf{A}$ is the MoM impedance matrix, $\mathbf{b}$ is the vector containing the incident field excitation, and $\mathbf{x}$ is the vector containing the unknown reflection coefficients. The matrix elements of $\mathbf{A}$ physically describe how a certain coaxial waveguide mode couples into another. For instance, the $\tilde{n}, 0$ element describes how the $0^{\text {th }}$, or TEM source (basis) mode couples into the $\tilde{n}^{\text {th }}$ field (testing) mode.

\section{RESULTS}

In this section, simulation results for the magnitude and phase of the reflection coefficient for a PEC-backed alumina sample $\left(d=5.5 \mathrm{~mm}\right.$ and $\left.\varepsilon_{r}=9, \mu_{r}=1\right)$ versus frequency and inner radius $a$ are presented. For the simulation, the coaxial line was assumed to be Teflon filled $\left(\varepsilon_{c}=2.1\right.$, $\mu_{c}=1$ ) and have a characteristic impedance of $50 \Omega$. The outer conductor $b$ was determined from the expression for $Z_{c}$, namely,

$$
Z_{c}=\frac{\eta_{c}}{2 \pi} \ln \left(\frac{b}{a}\right) .
$$

The simulated frequency band was chosen to be consistent with an Agilent E8362B vector network analyzer (VNA), i.e., $10 \mathrm{MHz}$ to $20 \mathrm{GHz}$ [15]. Three higher-order $\mathrm{TM}_{0 n}^{z}$ modes were considered in this analysis consistent with that performed in [6]. Last, to ensure only TEM propagation in the coax, the cut-off frequency of the $\mathrm{TE}_{11}^{z}$ mode was calculated by solving numerically

$$
J_{1}{ }^{\prime}\left(k_{\rho 1} a\right) Y_{1}{ }^{\prime}\left(k_{\rho 1} b\right)-J_{1}{ }^{\prime}\left(k_{\rho 1} b\right) Y_{1}{ }^{\prime}\left(k_{\rho 1} a\right)=0
$$

at each frequency and $a$. This cut-off frequency curve is included on the figures. Note that the primed Bessel functions in (5) represent derivatives with respect to their arguments. Two other low-loss MUTs, air and acrylic, were also simulated. Their reflection coefficient results are not included here for the sake of brevity.

Figure 3 shows the simulated reflection coefficient magnitude (Fig. 3a) and phase (Fig. 3b) results for the PECbacked alumina sample described above. The dashed curve on both figures is the cut-off frequency of the $\mathrm{TE}_{11}^{z}$ mode. The contours above and to the right of this curve cannot be used for design purposes because, in that region, multiple modes can propagate in the coaxial line. This region is marked by the label "Out of Operational Range" in the figures. Note that a large majority of the coaxial probe measurement results in the literature are for frequencies less than $6 \mathrm{GHz}$ [5], [6], [9], [10]. This corresponds to the lower right hand corner of the figures.

Inspection of Fig. 3 shows that the maximum bandwidth the coaxial probe will support is approximately $20 \mathrm{GHz}$ (vertical dashed-dotted line drawn on both figures) at $a=0.78 \mathrm{~mm}$.
However, the magnitude of the reflection coefficient $\left|S_{11}\right|$ remains greater than 0.95 from $10 \mathrm{MHz}$ to $8 \mathrm{GHz}$ implying poor field coupling into the MUT region of the probe for frequencies in this range. Note that a very similar behavior was observed for the air $\left(\left|S_{11}\right| \geq 0.95\right.$ for $\left.20 \mathrm{GHz}\right)$ and acrylic $\left(\left|S_{11}\right| \geq 0.95\right.$ for $11 \mathrm{GHz}$ ) simulation results (not shown). As discussed previously, this has important consequences for material characterization. Consider that the E8362B VNA $S_{11}$ magnitude and phase uncertainties vary (depending on calibration) between approximately $0.02-0.05$ and $2^{\circ}-4^{\circ}$, respectively [15]. Thus, it is not likely that one could determine via measurement the $\varepsilon_{r}$ of alumina with any accuracy until approximately $8 \mathrm{GHz}$ since its measured $S_{11}$ is "experimentally identical" to those of air and acrylic. Taking this reasoning into account, it seems that the maximum effective bandwidth is approximately $10 \mathrm{GHz}$, represented in the figures by green rectangles. This bandwidth is approximately $4 \mathrm{GHz}$ larger than that available using a $\mathrm{K}_{u}$-band (approximately $6 \mathrm{GHz}$ ) openended rectangular waveguide probe and several times larger than that reported in the literature for other coaxial probes.

To find the minimum flange size required with a $10 \mathrm{GHz}$ measurement bandwidth, the range resolution expression

$$
\Delta R=\frac{c}{2 B},
$$

where $c$ is the speed of light in vacuum and $B=10 \mathrm{GHz}$ is the bandwidth, is utilized. This expression yields a $\Delta R=1.5 \mathrm{~cm}$. This means that the flange of the open-ended coaxial probe can be reduced to approximately $1.5 \mathrm{~cm}$ in radius and the edge reflection (shown in Figs. 1 and 2) will still be resolved and thus can be effectively removed via time-domain gating [11]. Note that this flange size is significantly smaller than those used previously in the literature [6], [10].

\section{COnclusions \& Future Work}

In this paper, an open-ended coaxial probe was investigated in order to determine whether the geometry was sufficiently broadband to make it a good option as compared to a rectangular waveguide probe (previously investigated) and to present a design methodology which maximizes measurement bandwidth while limiting flange size (thus improving its applicability for nondestructive material characterization). This analysis was performed via a simulation which used Love's equivalence theorem and the parallel-plate Green's function to derive an MFIE which was subsequently solved for the reflection coefficient using the MoM. It was found that the maximum effective bandwidth of the probe is approximately $10 \mathrm{GHz}, 4 \mathrm{GHz}$ larger than the maximum bandwidth available using an open-ended rectangular waveguide probe. With this increased bandwidth, the size of the flange of the coaxial probe, calculated to be approximately $1.5 \mathrm{~cm}$ in radius, can be significantly reduced from what is typically reported in the literature.

Future work will include construction of the probe designed in this paper and experimental material characterization results. The methodology presented in this paper will also be used to design a two-port flanged coaxial probe similar to 


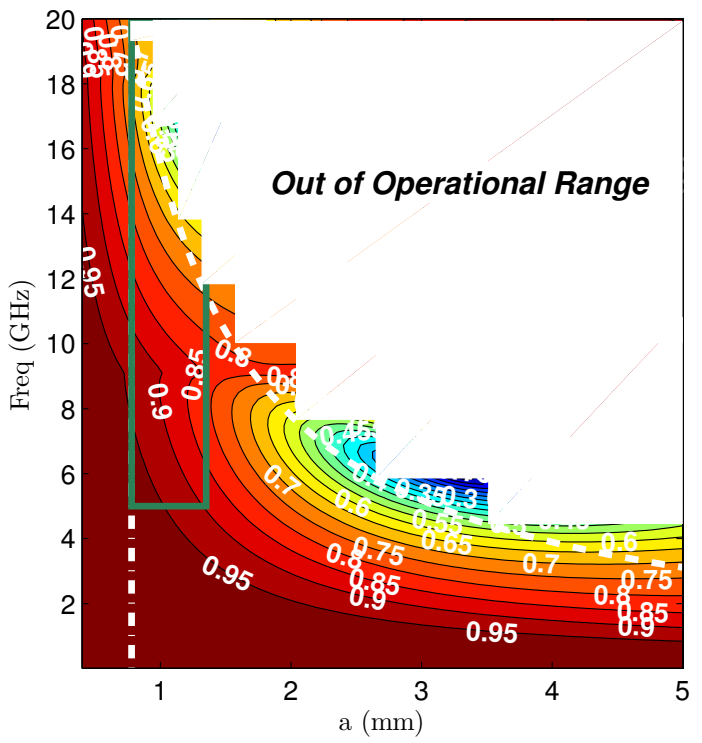

(a)

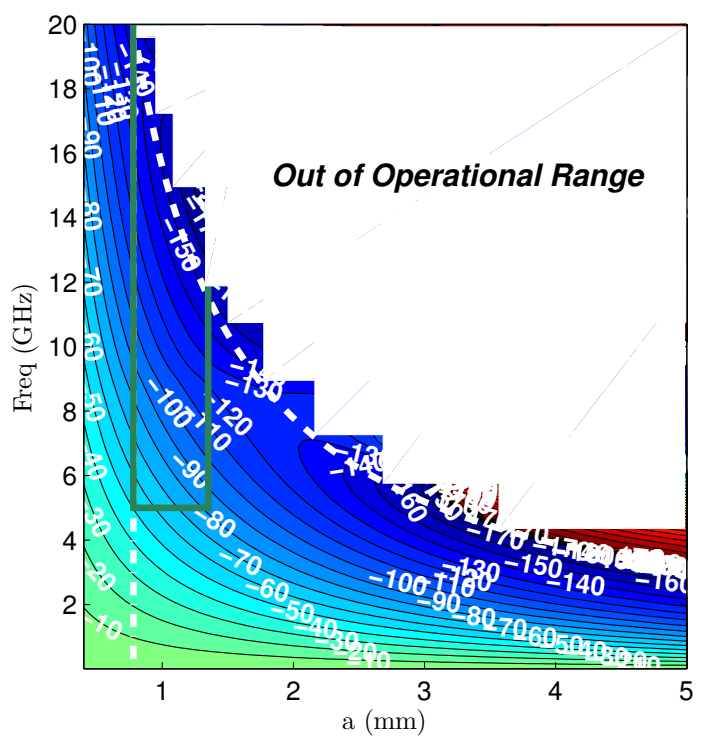

(b)

Fig. 3. (a) $\left|S_{11}\right|$ contour plot of alumina versus frequency and $a$. (b) $\angle S_{11}$ contour plot in degrees of alumina versus frequency and $a$. The white dashed curve is the cut-off frequency of the first higher-order coaxial waveguide mode. The $\left|S_{11}\right|$ and $\angle S_{11}$ results above and to the right of the cut-off frequency curves cannot be used for design purposes because, in that region, multiple modes can propagate in the coaxial line.

the one described in [16]. The two-port flanged coaxial probe will allow for the simultaneous collection of the reflection and transmission coefficients thus permitting the $\varepsilon_{r}$ and $\mu_{r}$ of the MUT to be determined unambiguously.

The views expressed in this paper are those of the authors and do not reflect the official policy or position of the U.S. Air Force, the Department of Defense, or the U.S. Government.

\section{REFERENCES}

[1] J.-Z. Bao, S.-T. Lu, and W. D. Hurt, "Complex dielectric measurements and analysis of brain tissues in the radio and microwave frequencies," IEEE Trans. Microwave Theory Tech., vol. 45, no. 10, pp. 1730-1741, Oct. 1997

[2] D. Popovic, L. McCartney, C. Beasley, M. Lazebnik, M. Okoniewski, S. C. Hagness, and J. H. Booske, "Precision open-ended coaxial probes for in vivo and ex vivo dielectric spectroscopy of biological tissues at microwave frequencies," IEEE Trans. Microwave Theory Tech., vol. 53, no. 5, pp. 1713-1722, May 2005.

[3] M. S. Boybay and O. M. Ramahi, "Open-ended coaxial line probes with negative permittivity materials," IEEE Trans. Antennas Propag., vol. 59, no. 5, pp. 1765-1769, May 2011.

[4] R. Olmi, M. Bini, R. Nesti, G. Pelosi, and C. Riminesi, "Improvement of the permittivity measurement by a 3D full-wave analysis of a finite flanged coaxial probe," J. Electromagn. Waves Appl., vol. 18, pp. 217-232, 2004. [Online]. Available: http://www.ingentaconnect.com/ content/vsp/jew/2004/00000018/00000002/art00011

[5] M. Wu, X. Yao, and L. Zhang, "An improved coaxial probe technique for measuring microwave permittivity of thin dielectric materials," Meas. Sci. Technol., vol. 11, no. 11, p. 1617, 2000. [Online]. Available: http://stacks.iop.org/0957-0233/11/i=11/a=311

[6] P. De Langhe, K. Blomme, L. Martens, and D. De Zutter, "Measurement of low-permittivity materials based on a spectral-domain analysis for the open-ended coaxial probe," IEEE Trans. Instrum. Meas., vol. 42, no. 5, pp. 879-886, Oct. 1993.

[7] S. Bakhtiari, S. Ganchev, and R. Zoughi, "Analysis of radiation from an open-ended coaxial line into stratified dielectrics," IEEE Trans. Microwave Theory Tech., vol. 42, no. 7, pp. 1261-1267, Jul. 1994.
[8] S. Ganchev, N. Qaddoumi, S. Bakhtiari, and R. Zoughi, "Calibration and measurement of dielectric properties of finite thickness composite sheets with open-ended coaxial sensors," IEEE Trans. Instrum. Meas., vol. 44, no. 6, pp. 1023-1029, Dec. 1995.

[9] J. Baker-Jarvis, M. Janezic, P. Domich, and R. Geyer, "Analysis of an open-ended coaxial probe with lift-off for nondestructive testing," IEEE Trans. Instrum. Meas., vol. 43, no. 5, pp. 711-718, Oct. 1994.

[10] C.-L. Li and K.-M. Chen, "Determination of electromagnetic properties of materials using flanged open-ended coaxial probe-full-wave analysis," IEEE Trans. Instrum. Meas., vol. 44, no. 1, pp. 19-27, Feb. 1995.

[11] M. W. Hyde, M. J. Havrilla, and A. E. Bogle, "A novel and simple technique for measuring low-loss materials using the two flanged waveguides measurement geometry," Meas. Sci. Technol., vol. 22, no. 8, p. 085704, 2011. [Online]. Available: http://stacks.iop.org/0957-0233/ $22 / \mathrm{i}=8 / \mathrm{a}=085704$

[12] R. E. Collin, Field Theory of Guided Waves, 2nd ed. New York, NY: IEEE Press, 1991.

[13] G. W. Hanson and A. B. Yakovlev, Operator Theory for Electromagnetics: An Introduction. New York, NY: Springer-Verlag, Inc., 2001.

[14] R. Harrington, Field Computation by Moment Methods. New York, NY: IEEE Press, 1993.

[15] Agilent Technologies, Technical Specifications Agilent Technologies PNA Series Network Analyzers E8362B/C, E8363B/C, and E8364B/C, Agilent Technologies, Oct. 2008.

[16] J. Baker-Jarvis and M. Janezic, "Analysis of a two-port flanged coaxial holder for shielding effectiveness and dielectric measurements of thin films and thin materials," IEEE Trans. Electromagn. Compat., vol. 38, no. 1, pp. 67-70, Feb. 1996. 